\title{
Design of Wireless Power Transmission for a Charge While Driving System
}

\author{
Davide Bavastro, Aldo Canova, Vincenzo Cirimele, Fabio Freschi, Luca Giaccone, Paolo Guglielmi, \\ and Maurizio Repetto \\ Department of Energy, Politecnico di Torino, Torino 10129, Italy
}

\begin{abstract}
The key components in wireless power transmission for the wireless recharging of electric vehicles are the pair made by the external power line and the on-board pickup module. This paper analyzes a charge while driving system by using a lumped parameter extraction with the partial element equivalent circuit method and a circuit simulation.
\end{abstract}

Index Terms-Charge while driving (CWD), fully electric vehicle, partial element equivalent circuit.

\section{INTRODUCTION}

C LEAN transportation represents a key economic and social contribution to the development of modern and future society. Hybrid electric vehicles and full electric vehicles (FEVs) presence is fast increasing in agreement with the oil price rising and a stronger sensitivity to environmental issue. One of the principal and more critical aspect in the FEVs marketplace diffusion is the battery recharge system: users expect to be assisted in real time and without long waiting times.

The wireless power transfer (WPT) for the charging of electric vehicles was supposed to be only an academic technology without any commercial possibility. Owing to the growth of the market of electric vehicles and wireless technology, many electric vehicle manufacturers have shown their interest into the wireless charging of electric vehicles. The static wireless charging is now a common technique proposed by many manufacturers. The charger consists basically of two parts: 1) a charging plate attached to the bottom of the vehicle and 2) a charging mat placed on, or below, the ground. The efficiencies can go up to $90 \%$. The new frontiers of research in WPT is the so-called charge while driving (CWD) system: the goal is to extend the battery range by a fast partial recharging during the movement of the vehicle. The attention of manufacturers in wireless charging systems is also witnessed by an increasing number of recent papers dealing with this technology [1], [2].

This paper focuses the attention on the design of the inductive power transfer system by using the partial element equivalent circuit method to extract the lumped parameters used in a circuit simulator.

\section{ECO-FEV PROJECT}

The EU project efficient Cooperative infrastructure for Fully Electric Vehicles (eCo-FEV) ${ }^{1}$ co-funded by the European Union 7th Framework Programme for Research, aims at

Manuscript received June 29, 2013; accepted September 12, 2013. Date of current version February 21, 2014. Corresponding author: F. Freschi (e-mail: fabio.freschi@polito.it).

Color versions of one or more of the figures in this paper are available online at http://ieeexplore.ieee.org.

Digital Object Identifier 10.1109/TMAG.2013.2283339

${ }^{1}$ http://www.eco-fev.eu

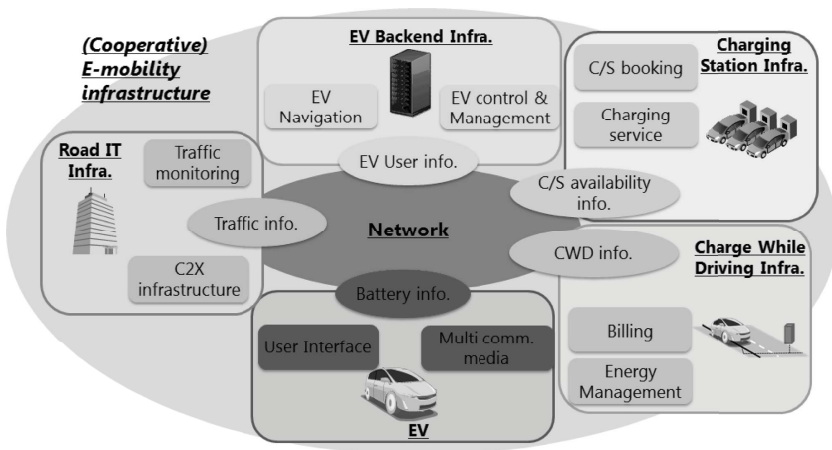

Fig. 1. Background overview of systems involved in the eCo-FEV project.

achieving a breakthrough for FEV introduction. It proposes an information technology (IT) electric mobility platform for the integration of FEV into infrastructures, enabling connections and information exchanges between multiple infrastructure systems, such as road IT, FEV backend, and charging infrastructure. Under this framework, one of the interests of the project is the design, development, and performance analysis of static and dynamic wireless FEV charging capabilities.

By making the infrastructure systems and FEVs cooperate with each other, advanced telematics services for FEV users will be enabled, allowing FEV users to quickly react on the driving situations and efficiently manage the charging during the whole travel, in the meanwhile trying to satisfy the traveling needs (e.g., arrival time) and economic needs (e.g., charging cost) of FEV users. An overview of systems involved in the eCo-FEV project and their cooperation is shown in Fig. 1.

Multiple stakeholders are included in the eCo-FEV consortium to evaluate in real sites the telematics services and potential business models, including car manufacturers, traffic management operator, system provider, FEV service provider, energy trader, and universities. The project will provide technical solutions for some standardization gaps, in particular, wireless communication between EV and operator and wireless charging.

\section{PROBLEM DESCRIPTION}

The aim of this paper is to prove the feasibility of an iron less WPT system for the recharging of a light commercial 


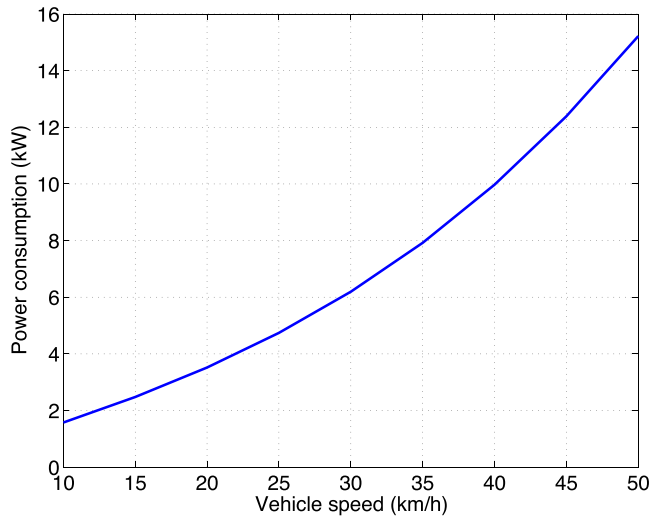

Fig. 2. Power versus constant speed.

electric vehicle during the motion along a specific road. The choice of the iron less technology is due to the fact that the recharging system must be added to an existing electric vehicle with a low impact on the original layout.

The power rating of the WPT system is chosen to fulfill the power consumption of the vehicle. A simple steady-state model of motion is considered here

$$
F_{m}=m g\left(C_{r} \cos (\varphi)+\sin (\varphi)\right)+\frac{1}{2} \rho_{\text {air }} A_{f} C_{x} v^{2}
$$

where $F_{m}$ is the motive force, $C_{r}$ is the friction coefficient, $\varphi$ is the road slope, $\rho_{\text {air }}$ is the air density, $A_{f}$ is the estimated frontal area of the vehicle, $C_{x}$ is the drag coefficient, and $v$ the vehicle speed. A total efficiency of electric motor and transmission system of $\eta=0.8$ and a mass of $3500 \mathrm{~kg}$ are assumed. A constant speed is assumed during the wireless recharge. The power supplied by the batteries is then

$$
P_{\text {batt }}=\frac{1}{\eta} F_{m} v
$$

and is shown in Fig. 2. To ensure the frequency regulation and to dynamically maintain a stable power transmission, it is convenient to limit the speed of the vehicle to $30 \mathrm{~km} / \mathrm{h}$. A system with power rating of $20 \mathrm{~kW}$ is chosen to ensure that the power transmitted to the vehicle is sufficient to cover the self-consumption and, at the same time, recharge the batteries.

\section{System ARchitecture}

A thorough analysis of the literature shows that most WPT systems use current-controlled structures. This choice is justified by the high-current level on the primary side and the possibility to regulate the transferred power by regulating the current.

In this paper, a voltage source inverter solution using MOSFETs is adopted (see Fig. 3). This makes it possible to have a fast regulating system capable of responding to the rapid change of the inductive system parameters during the vehicle motion and a higher switching frequency for the power transmission. The use of MOSFETs allows a larger frequency range $(20-200 \mathrm{kHz}$ in this case) with lower switching losses with respect to the traditional systems using IGBTs.

The power electronics works with a symmetrical square voltage waveform. The regulation of the transmitted power

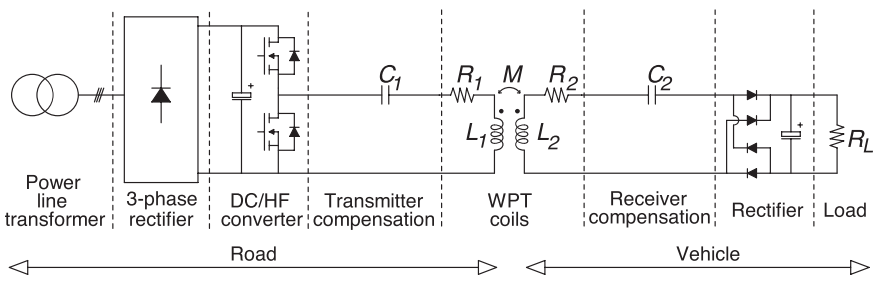

Fig. 3. Architecture of the CWD system.

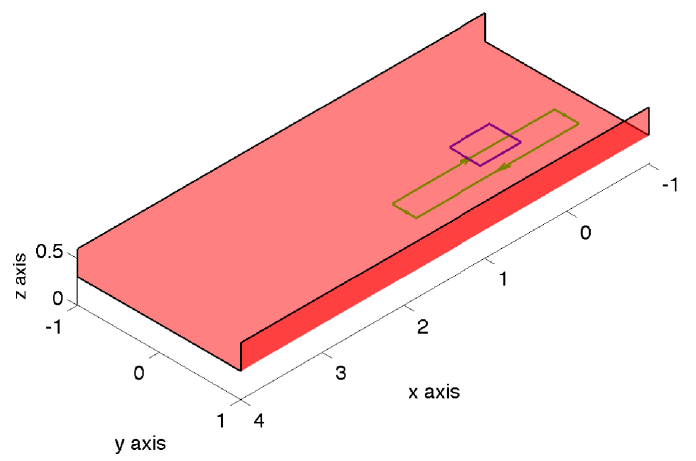

Fig. 4. Schematic representation of the CWD system under study.

is achieved by the frequency tuning because the regulation of the peak voltage is not advisable. As showed in [3], system stability condition implies to have a quality factor of the primary side $Q_{p}$ higher than the secondary one $Q_{s}$.

\section{PReliminaries}

The domain under study is made by the coils and part of the car body that represents the vehicle (see Fig. 4). The resonant frequency of the system is set to $100 \mathrm{kHz}$. A preliminary study showed that at this frequency, the magnetic properties of the car body are not significant and they are neglected in the study. The penetration depth is $20 \mu \mathrm{m}$, so that the conductive domain can be suitably modeled as a 3-D surface.

The system is characterized by three coupled circuits: emitter coil, receiver coil, and the conductive domain. A proper modeling would consider the three linked fluxes

$$
\begin{aligned}
& \lambda_{1}=L_{11} i_{1}+L_{12} i_{2}+L_{13} i_{3} \\
& \lambda_{2}=L_{21} i_{1}+L_{22} i_{2}+L_{23} i_{3} \\
& \lambda_{3}=L_{31} i_{1}+L_{32} i_{2}+L_{33} i_{3}
\end{aligned}
$$

where the third contribution is due to the eddy currents. This implies a complete field-circuit coupling as proposed, for example, in [4]. In spite of the fact that the eddy currents significantly change the field distribution, influencing the value of the mutual inductances, it has been verified that the value of the linked flux is weakly influenced by them, for example, considering only the first coil active

$$
\begin{aligned}
& L_{11}^{\prime}=\frac{\lambda_{1}}{i_{1}}=L_{11}+L_{13} \frac{i_{3}}{i_{1}} \\
& L_{21}^{\prime}=\frac{\lambda_{2}}{i_{1}}=L_{21}+L_{23} \frac{i_{3}}{i_{1}} \\
& L_{31}^{\prime}=\frac{\lambda_{3}}{i_{1}}=L_{31}+L_{33} \frac{i_{3}}{i_{1}}
\end{aligned}
$$


with $i_{3} / i_{1} \rightarrow 0$. Thus, the operative inductances $L_{11}^{\prime}$ and $L_{21}^{\prime}$ (as well as $L_{22}^{\prime}$, calculated in the same way) will be considered in the circuit simulation.

\section{Dual-PEEC Formulation and Partial ELEMENT CALCULATION}

Owing to the peculiarities of the problem, a surface dualPEEC formulation is adopted for the simulations.

The total electric field, which is at the observation point $\vec{r}$ and angular frequency $\omega$, takes the form

$$
\frac{\vec{J}(\vec{r}, \omega)}{\kappa}+\mathrm{j} \omega \vec{A}(\vec{r}, \omega)+\nabla \varphi(\vec{r}, \omega)=0
$$

where $\kappa$ is the electric conductivity, $\vec{J}$ is the current density, and $\varphi$ is the electric scalar potential. The magnetic vector potential $\vec{A}$ is expressed as

$$
\vec{A}(\vec{r}, \omega)=\frac{\mu}{4 \pi} \int_{\Omega} \frac{\vec{J}\left(\vec{r}^{\prime}, \omega\right)}{\left|\vec{r}-\vec{r}^{\prime}\right|} \mathrm{d} \Omega
$$

where $\vec{r}^{\prime}$ is the source point and $\Omega$ is the conductive domain.

The dual-PEEC method is a generalization of the PEEC for unstructured triangular tessellations [5], [6]. The main distinction from the standard PEEC technique is the use of two grids of interlocked cells, called primal and dual discretizations [7]. Duality relations hold between the primal and dual-cell complex: 1) primal nodes correspond to dual volumes; 2) primal edges to dual faces; 3 ) primal faces to dual edges; and 4) primal volumes to dual nodes. In accordance with the work of Tonti [8], each variable is strictly associated to a spatial element: electric potentials are assigned to the barycenters of triangular discretization, voltages to the edges connecting these nodes, currents flow through the edges of triangles, while electric charges are defined on the triangles.

The current density is expanded with facet elements $\vec{w}_{k m}$

$$
\vec{J}_{m}=\frac{1}{\delta_{m}} \sum_{k=1}^{3} \vec{w}_{k m} i_{k m}
$$

where $i_{k m}$ is the electric current flowing along the $k$ th edge of triangle $m$. Basis functions (10) are substituted into (9) discretized over the $m$ th triangle

$$
\begin{aligned}
& \frac{1}{\delta_{m} \kappa_{m}} \sum_{k=1}^{3} \vec{w}_{k m}(\vec{r}) i_{k m}(\omega) \\
& \quad+\frac{\mathrm{j} \omega \mu}{4 \pi} \sum_{m} \int_{\Sigma_{m}} \frac{1}{\left|\vec{r}-\vec{r}^{\prime}\right|} \sum_{k=1}^{3} \vec{w}_{k m}\left(\vec{r}^{\prime}\right) i_{k m}(\omega) \mathrm{d} \Sigma \\
& \quad+\nabla \varphi(\vec{r}, \omega)=0 .
\end{aligned}
$$

The same bases are then used as test functions in a Galerkin scheme [9]. The expansion of (11) gives rise to a resistive and inductive contribution

$$
\begin{aligned}
R_{k m, h m} & =\frac{1}{\delta_{m} \kappa_{m}} \int_{S_{m}} \vec{w}_{k m} \cdot \vec{w}_{h m} \mathrm{~d} S_{m} \\
L_{k m, h n} & =\frac{\mu_{0}}{4 \pi} \int_{S_{m}} \vec{w}_{k m} \cdot \int_{S_{n}} \frac{\vec{w}_{h n}}{\left|\vec{r}-\vec{r}^{\prime}\right|} \mathrm{d} S_{n} \mathrm{~d} S_{m} .
\end{aligned}
$$

The partial inductance coefficients require the computation of singular integrals, having $\vec{w}_{h n} /\left|\vec{r}-\vec{r}^{\prime}\right|$ as kernel, over

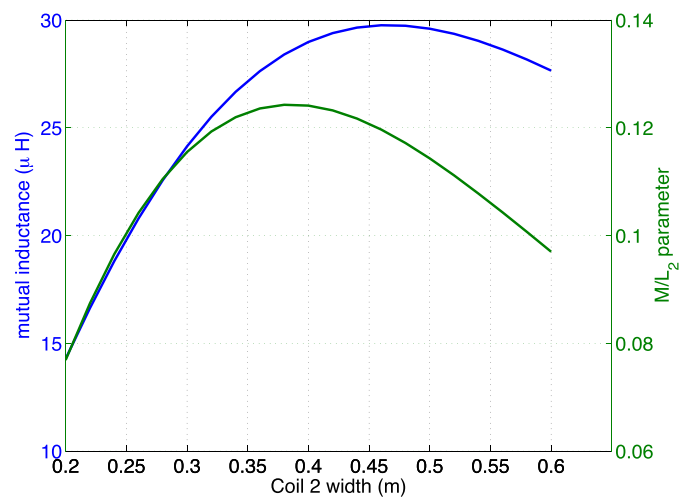

Fig. 5. $M$ and $M / L_{2}$ versus receiving coil width.

TABLE I

INDUCTIVE PARAMETERS

\begin{tabular}{lccc}
\hline Param & $\begin{array}{c}\text { 3D } \\
\text { w/ carbody }\end{array}$ & $\begin{array}{c}\text { 2D } \\
\text { w/ carbody }\end{array}$ & Analytic \\
\hline$L_{1}$ & $3.0 \mathrm{mH}$ & $0.5 \mathrm{mH}$ & $1.4 \mathrm{mH}$ \\
$L_{2}$ & $452.8 \mu \mathrm{H}$ & $49.3 \mu \mathrm{H}$ & $229.8 \mu \mathrm{H}$ \\
$M$ & $22.1 \mu \mathrm{H}$ & $28.8 \mu \mathrm{H}$ & $35.9 \mu \mathrm{H}$ \\
\hline
\end{tabular}

triangular domains. Analytical formulas exist for the solution of the inner integral [10]. The outer integral is computed numerically by a 16-point quadrature rule on the triangle (eighth order quadrature rule). Owing to the use of analytical formulas for the inner integral, the numerical quadratures do not have to cope with any singularity. Thus, the theoretical accuracy is guaranteed also in the computation of the selfterms. The integration routines have been parallelized with OpenMP at the coarsest level, i.e., all triangular elements are processed in parallel.

\section{RESULTS}

\section{A. Coil Geometry}

The inductive transmission system is based on loosely coupled inductors. The dimensions of the pickup coil are chosen to maximize the ratio $M / L_{2}$ with a fixed dimension of the transmitter coil. Consequently, the voltage drop across the pickup inductance is minimized, as well as the primary current necessary to transfer the desired power.

It is worth noting that the maximum $M / L_{2}$ is different from the maximum mutual inductance, as shown in Fig. 5. This maximum is obtained with a width of the received coil of $39 \mathrm{~cm}$ when the width of transmitting coil is $30 \mathrm{~cm}$. The number of turns is 22 and 14 for the primary and secondary coils, respectively.

\section{B. Circuit Parameters}

The field analysis allows the extraction of the self- and mutual inductance parameters, according to the assumptions described in the previous sections. The values that correspond to the optimal sizing of Fig. 5 are reported in Table I. The 3-D simulations give different results from the analytical 
TABLE II

SimUlation PARAMETERS

\begin{tabular}{lc|lc}
\hline parameter & value & parameter & value \\
\hline$L_{1}$ & $1.4 \mathrm{mH}$ & $R_{1}$ & $240 \mathrm{~m} \Omega$ \\
$L_{2}$ & $229.8 \mu \mathrm{H}$ & $R_{2}$ & $60 \mathrm{~m} \Omega$ \\
$\mathrm{M}$ & $22.1 \mu \mathrm{H}$ & $R_{L}$ w/o bridge & $12 \Omega$ \\
$C_{1}$ & $1.8 \mathrm{nF}$ & $R_{L}$ w/ bridge & $15 \Omega$ \\
$C_{2}$ & $11 \mathrm{nF}$ & & \\
\hline
\end{tabular}
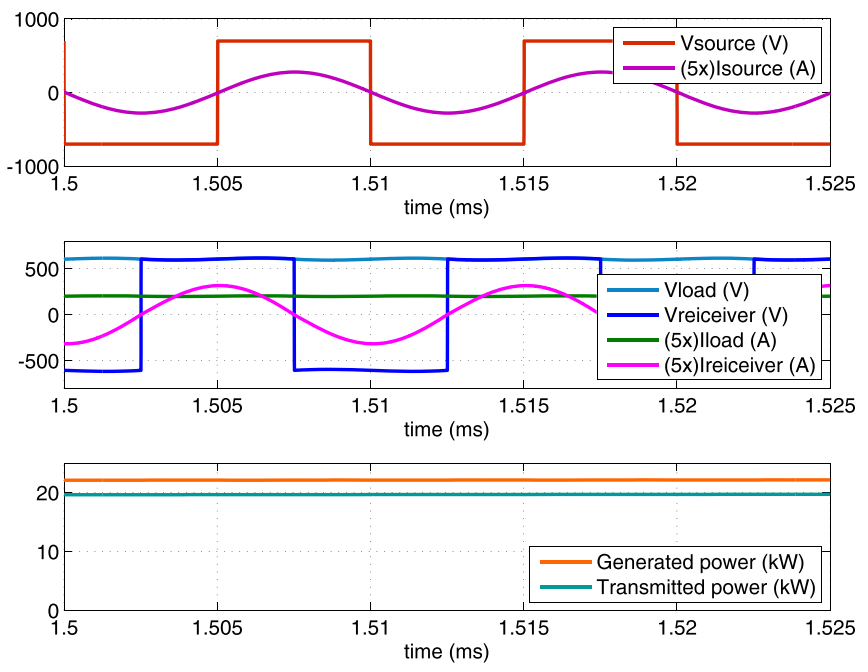

Fig. 6. Primary and secondary coil waveforms.

formulas [11] and 2-D simulations. While 2-D simulations are not reliable in this case, due to the oversimplification of the geometry, the analytic values of self-inductances seem to be more accurate than the 3-D simulations. In the latter case, in fact, filamentary coils are considered, neglecting the internal inductance of the coil itself. This fact is less important for the calculation of mutual inductances. Therefore, the circuit simulations are run using the analytical value of the selfinductance and the numerical value of mutual inductance obtained by 3-D simulation.

According to the procedure suggested in [3], with these values it is also possible estimate the compensation capacitances, the stability factor, and the power transfer capability of the system. Compensation capacitances are inserted to reach system resonance. On the power electronic side, resonance allows to adopt a zero-voltage-zero-current switching converter reducing ideally to zero the switching losses. The values of components used for the circuit simulation are shown in Table II. The value of the load resistance is chosen to guarantee the power transmission with a good efficiency and tolerable values of output current. This choice is possible because of the use of $\mathrm{dc} / \mathrm{dc}$ converter for load adaptation. In case of presence of a rectifier, made of a diode bridge with filter capacitance, it is necessary to adopt an other value for the resistor $R_{L}$ using a coefficient equal to $\pi^{2} / 8$.

\section{Analysis of the Complete System}

Results in Fig. 6 show that the resonance condition is achieved both at the emitter and in the receiver section. The efficiency of the power transmission is about $90 \%$ and the reactive power generated is nearly zero. This means that the desired zero-voltage and zero-current condition is reached. Simultaneously, in resonance condition, the effect of the harmonics of the square voltage waveform is negligible because of the high-quality factor. The resultant current waveform is sinusoidal.

\section{ACKNOWLEDGMENT}

This work was supported in part by the European Unions 7th Framework Programme for Research, namely through the STREP eCo-FEV project FP7 314411.

\section{REFERENCES}

[1] S. Ho, J. Wang, W. Fu, and M. Sun, "A comparative study between novel witricity and traditional inductive magnetic coupling in wireless charging," IEEE Trans. Magn., vol. 47, no. 5, pp. 1522-1525, May 2011.

[2] J. Wang, S. Ho, W. Fu, and M. Sun, "Analytical design study of a novel witricity charger with lateral and angular misalignments for efficient wireless energy transmission," IEEE Trans. Magn., vol. 47, no. 10, pp. 2616-2619, Oct. 2011.

[3] C. S. Wang, G. Covic, and O. Stielau, "Power transfer capability and bifurcation phenomena of loosely coupled inductive power transfer systems," IEEE Trans. Ind. Electron., vol. 51, no. 1, pp. 148-157, Feb. 2004.

[4] O. Bottauscio, A. Manzin, A. Canova, M. Chiampi, G. Gruosso, and M. Repetto, "Field and circuit approaches for diffusion phenomena in magnetic cores," IEEE Trans. Magn., vol. 40, no. 2, pp. 1322-1325, Mar. 2004.

[5] F. Freschi, G. Gruosso, and M. Repetto, "Unstructured PEEC formulation by dual discretization," IEEE Microw. Wireless Compon. Lett., vol. 16, no. 10, pp. 531-533, Oct. 2006.

[6] F. Freschi, G. Gruosso, A. Maffucci, M. Repetto, F. Villone, and W. Zamboni, "Investigation of low-frequency behaviour of two surface integral full-Maxwell algorithms," Int. J. Comput. Math. Electr. Electron. Eng., vol. 26, no. 3, pp. 842-858, 2007.

[7] F. Freschi and M. Repetto, "A general framework for mixed structured/unstructured PEEC modelling," ACES J., vol. 23, no. 3, pp. 200-206, Sep. 2008.

[8] E. Tonti, "Finite formulation of electromagnetic field," IEEE Trans. Magn., vol. 38, no. 2, pp. 333-336, Mar. 2002.

[9] P. Alotto, D. Desideri, F. Freschi, A. Maschio, and M. Repetto, "DualPEEC modeling of a two-port TEM cell for VHF applications," IEEE Trans. Magn., vol. 47, no. 5, pp. 1486-1489, May 2011.

[10] R. Graglia, "On the numerical integration of the linear shape functions times the 3-D Green's function or its gradient on a plane triangle," IEEE Trans. Antennas Propag., vol. 41, no. 10, pp. 1448-1455, Oct. 1993.

[11] J. Sallán, J. Villa, A. Llombart, and J. Sanz, "Optimal design of ICPT systems applied to electric vehicle battery charge," IEEE Trans. Ind. Electron., vol. 56, no. 6, pp. 2140-2149, Jun. 2009. 